\title{
The Influence of Fuel Pressure Ripple on Performances of Engine Control System
}

\author{
V. Shorin, A. Gimadiev and V. Sverbilov \\ Samara State Aerospace University, Samara, Russian Federation \\ E-mail: aseu@ssau.ru, gimadiev_ag@mail.ru, v.sverbilov@mail.ru
}

\begin{abstract}
Pressure and flow ripple generated by a fuel pump have essential impact on static and dynamic performances of an engine control system. It is especially dangerous for open-loop control used at a turbojet starting mode. The fuel consumption deviation at the engine starting caused by nonlinear averaging of fluctuations over throttling elements can reach up to $25 \%$ of a nominal value and provoke engine stopping. In the paper, the method for calculation of the hydro mechanical system characteristics in the presence of fluid born noise and its application for the turbojet start control system are developed. The harmonic analysis and the least mean square (LMS) method for the restrictor performance linearization is used. To avoid influence of fuel flow disturbances on the engine static performance it is proposed to increase system accuracy at the starting mode by placing the hydraulic $R L$-filter into the control unit. The effect of the device was surely verified by the engine test results.
\end{abstract}

Keywords: Fuel flow ripple, turbojet control system, performance accuracy

\section{Introduction}

The problem of high flow ripple level generated in hydraulic systems is well known [e.g., 1]. The fluctuations of a working fluid caused by non-uniformity in delivery of hydraulic and fuel pumps, instability of separate system circuits, have essential impact on static and dynamic performances of aircraft power and control systems. Working fluid ripple is especially dangerous for turbojet open-loop control systems at a start mode. The fuel consumption deviation at the engine starting caused by nonlinear averaging of fluctuations across resistive elements can reach up to $25 \%$ of a nominal value. Therefore research of characteristics of engine control systems taking into account high-frequency noise to develop measures for increasing their static and dynamic accuracy is very important and actual.

The problem of calculation of control system static and dynamic characteristics in the presence of fluid born noise is generally reduced to searching of the solution of system of the nonlinear differential equations with variable parameters. Variable parameters are hydraulic resistances of throttling elements evaluated as a result of solution of an equation subsystem in relation to high-frequency fluctuations. Such decision is possible at an assumption that a characteristic time of dynamic process of a desired signal is much more (at least by $5 . . .10$ times) than the greatest period of oscillation components.

In the paper, the generalized scheme of a turbojet control system containing in its structure sources of fluid oscillations is considered. The fluctuation sources are presented in the form of equivalent flow sources, the system parameters - in the form of the sums of slow variable and high-frequency components.

The calculation models of the throttling elements are presented separately for a slow useful signal and a highfrequency noise in the form of two interconnected equation systems. The high-frequency model of the control system is considered as distributed parameters system with circuit elements described in the form of acoustic two-port network. The system parts containing moving elements are presented in the form of six-pole network, which matrix coefficients depend on the frequency of fluctuations, parameters of connecting channels and damping properties of the element.

The simultaneous solution of the nonlinear differential and algebraic equations is made by a numerical method. On an initial step of iterations the slowly changing system parameters are calculated without high-frequency fluctuations, then, having used an impedance method, complex amplitudes of pressure/flow oscillations in the allocated sections and pressure drops across restrictors are determined. The calculation by such iterative method is conducted till ensuring the required accuracy.

The realization of the stated technique is presented to estimate influence of high-frequency fluctuations of a working fluid on static performances of the turbojet at the starting mode and to develop measures for increase system accuracy under conditions of fuel flow pulsations. 


\section{Modeling}

Consider hydraulic circuit having some source of FBN, which can be presented as equivalent flow source

$$
Q(t)=Q_{0}+\sum_{r=1}^{r=R} q_{r} \exp \left(j \omega_{r} t\right)
$$

where $Q_{0}$ - steady flow component; $q_{r}$ - complex amplitude of flow rate for $r$-component of spectrum; $\omega_{r}-$ circular frequency of $r$-component; $r=1 \ldots R$-harmonic number.

Then pressure, flow rate at a cross section $i j$ and displacement of a moving element $n$ of the circuit can be presented by sums of slow varying and high-frequency components

$$
\left.\begin{array}{l}
P_{i \gamma}(t)=P_{i, 0}+\sum_{r=1}^{r=R} p_{i \gamma}^{(r)} \exp \left(j \omega_{r} t\right) ; \\
Q_{i \gamma}(t)=Q_{i, 0}+\sum_{r=1}^{r=R} q_{i \gamma}^{(r)} \exp \left(j \omega_{r} t\right) ; \\
H_{n}(t)=H_{n, 0}+\sum_{r=1}^{r=R} h_{n}^{(r)} \exp \left(j \omega_{r} t\right),
\end{array}\right\}
$$

where $P, Q$ and $H$ - corresponding pressure, flow rate and displacement; $i=1 \ldots I$ - number of circuit segment; $\gamma=1$ corresponds to inlet of circuit segment; $\gamma=2$ - corresponds to outlet of circuit segment; $n=1 \ldots N$ - number of moving element.

Since the control system nonlinear properties are mainly defined by throttling elements, for calculation of the poly harmonic oscillatory process it is expedient to use harmonic analysis and the least mean square (LMS) method for the throttle performances linearization.

Model of hydraulic resistance $k$ can be presented according to (2) for a slow varying useful signal by

$$
\Delta P_{k}=L_{k} \frac{d Q_{k}}{d t}+\varsigma_{\ln , k} Q_{k}+\varsigma_{n \ln , k}\left(Q_{k}-Q_{k r, k} \operatorname{sign} Q_{k}\right)^{2} \operatorname{sign} Q_{k}+\delta P_{k}
$$

and for high frequency components by

$$
\Delta p_{k}^{(r)} e^{j \omega_{r} t}=j L_{k} \omega_{r} q_{k}^{(r)} e^{j \omega_{r} t}+b_{k} q_{k}^{(r)}
$$

where $\Delta P_{k}$ - slow varying component over nonlinear resistance $k ; L_{k}$ - inductance of throttle element; $\varsigma_{\ln , k}, \zeta_{n \ln , k}$ - coefficients of linear and non linear hydraulic loses over throttling element; $Q_{k r, k}$ - critical flow rate till linear law of hydraulic loses exists; $\delta P_{k}$ - pressure drop increment over throttle element due to non linear averaging of pressure fluctuations; $\Delta p_{k}^{(r)}, q_{k}^{(r)}$ - complex amplitudes of pressure drop and flow rate over throttling element $k$ for a spectrum component $r ; b_{k}$ - linearization coefficient for pressure/flow curve of the throttle $k$.
Linearization coefficient $b_{k}$ and shift function (or systematic error) $\delta P_{k}$ are determined using LMS method (flow oscillation as input signal).

A typical system part where the mean pressure shift occurs is presented in Figure 1.

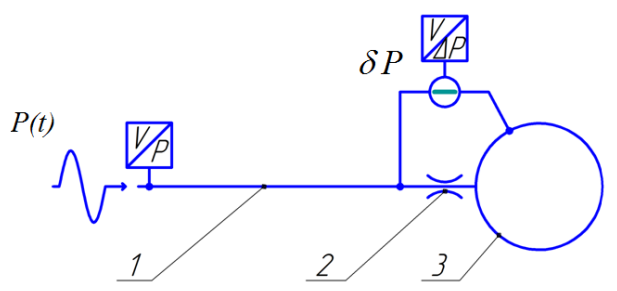

Figure 1: Diagram of a lateral pipeline: 1 -pipe $(d=7.8 \mathrm{~mm}$, $L=3.3 \mathrm{~m}$ ), 2 - restrictor (an orifice plate) $d=1.04 \mathrm{~mm}, 3-$ vessel $V=0.5 l$

A restrictor 2 is located close to capacity input 3 at the pipe end. The restrictor has a sharp front edge and a rear edge angle $120^{\circ}$. Working fluid viscosity is $20^{*} 10^{-6} \mathrm{~m}^{2} / \mathrm{s}$, density is $780 \mathrm{~kg} / \mathrm{m}^{3}$.

An input signal

$$
P(t)=P_{0}+p_{1} \exp \left(j \omega_{1} t\right)
$$

where $P_{0}=40 \mathrm{bar}, p_{1}=4.2 \mathrm{bar}$.

Theoretical and experimental research shows that pressure oscillations close to harmonic signal give a constant component of pressure difference over such asymmetrical restrictor (Figure 2). The similar phenomenon is observed at polyharmonic oscillations so over symmetric as asymmetric restrictors.

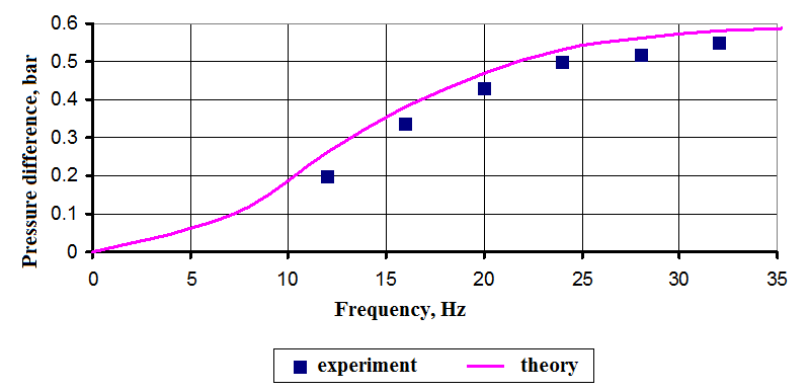

Figure 2: Dependence of a constant component of pressure difference on the frequency of fluctuations

Thus, if hydraulic system has a closed volume with a throttling element at the input, pressure pulsations can lead to emergence of pressure difference on a throttle which value changes depending on a spectrum of pulsations. Thus mean pressure in the cavity will drift relatively mean pressure in the system. In some systems it can have critical consequences.

\section{Application example}

Efficiency of the outlined method can be demonstrated on the example of turbojet control unite shown in Figure 3 pressure ripple being generated by fuel gear pump 4 . 


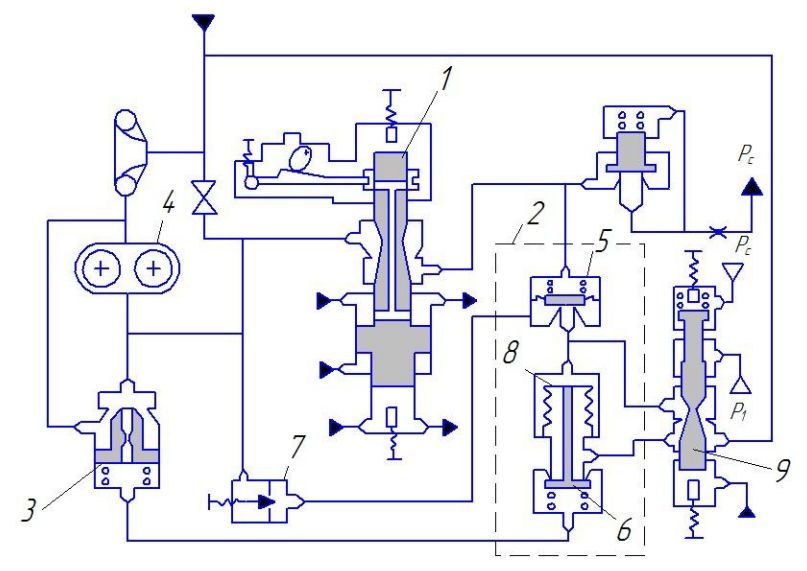

Figure 3: Diagram of the engine fuel control unit: 1metering pin; 2-pressure drop control valve; 3-pressure relief valve; 4-gear pump; 5-sensing element (pilot valve);

6-slave valve; 7-throttle; 8-bellow; 9-pressure drop compensator

At a starting mode of operation a desired fuel rate determines by a control handle and a compressor pressure ratio. Fuel variation is carried out by moving metering pin 1 and changing its flow area. The pressure drop across the metering pin 1 is stabilized by the control valve 2 acting on the relief valve 3 . If pressure drop across the pin 1 increases then the sensing element 5 moves upwards causes the valve 6 to open and to lower pressure in the spring chamber of the relief valve 3 . The latest goes down increasing by-pass flow from the pump output to the input thus recovering pressure differential across the pin 1 and the fuel flow rate too.

The fuel flow rate must increase with the compressor pressure ratio $P_{c} / P_{l}{ }^{*}$ increasing. This task is carried out by the pressure drop compensator 9 . As the ratio $P_{c} / P_{l}{ }^{*}$ increases, the needle valve of the compensator 9 moves down in Figure 3, thus increasing the fuel discharge flow from the chamber outside bellow 8 leading to the valve 6 closing. Then pressure in the spring chamber of the valve 3 increases, the latest moves upwards closing flow area, thus increasing pressure at the input of the metering pin 1 that consequently results in the fuel flow $Q_{d}$ increase.

In the case of the fuel overflow the protection system can interrupt engine starting because of thermal overload. The possible reason of the excess fuel flow can be an increase of the pressure drop across the metering pin 1 as a result of non linear averaging of pressure fluctuation generated by the pump 4 over the restrictor 7 . It leads to an unwarranted change of pressure drop over the membrane 5 and consequently to changes in pressure drop across the metering pin 1 and the fuel flow rate.

It was shown in the present research that the fuel flow deviation depends on a speed of the pump rotation, amplitudes of flow ripple spectral components and on the structure parameters of the pipeline between the pump and the controller, volumes and passages of the control unit, resistances of the pin 1 and the throttle 7 and their asymmetry. At the engine starting the most fuel flow deviation occurs at the rotor speed 5000 RPM and 7600 RPM. At these rotations flow ripple reaches the most intensity, maximum pressure drop is realized across the restrictor 7 and the pin 1, consequently resulting in maximum drift of fuel flow rate from the desired value (see Figure 4).

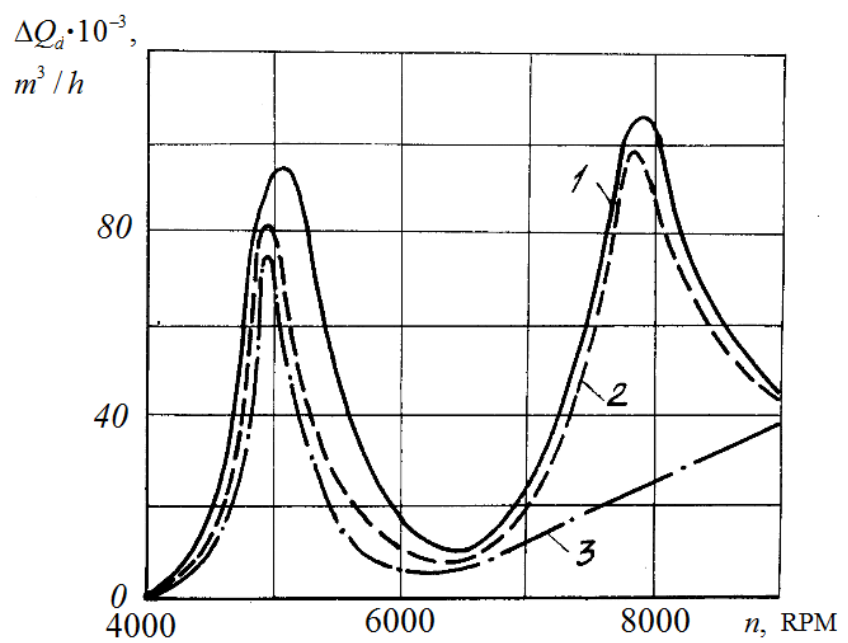

Figure4: Fuel flow rate fluctuations at the outlet of the metering pin for various spectrum of flow ripple at the pump outlet: $1-A_{q}^{(1)}=0.125 Q_{0}, A_{q}^{(2)}=0.5 A_{q}^{(1)}, A_{q}^{(3)}=0.3 A_{q}^{(1)}$;

$$
2-A_{q}^{(1)}=0.125 Q_{0}, A_{q}^{(2)}=0.5 A_{q}^{(1)} ; 3-A_{q}^{(1)}=0.125 Q_{0}
$$

The paper presents some results of analysis of the impact of the structure parameters and the spectral composition on the mean fuel flow rate deviation.

To suppress flow ripple and thus decrease the fuel flow deviation it was proposed to use an acoustic filter. Such approach is well known and described [e.g., 2, 3 and 4]. The acoustic $R L$-filter was placed in the line of the restrictor 7 . The filter presents a composition of hydraulic resistive and inductive elements. The basic issues of the filters and silencers design and application are considered in [2, 5]. Its parameters were determined on the base of a sizing procedure provided avoidance of the flow ripple influence on the engine start response. Experimental results of its application are shown in Figure 5.
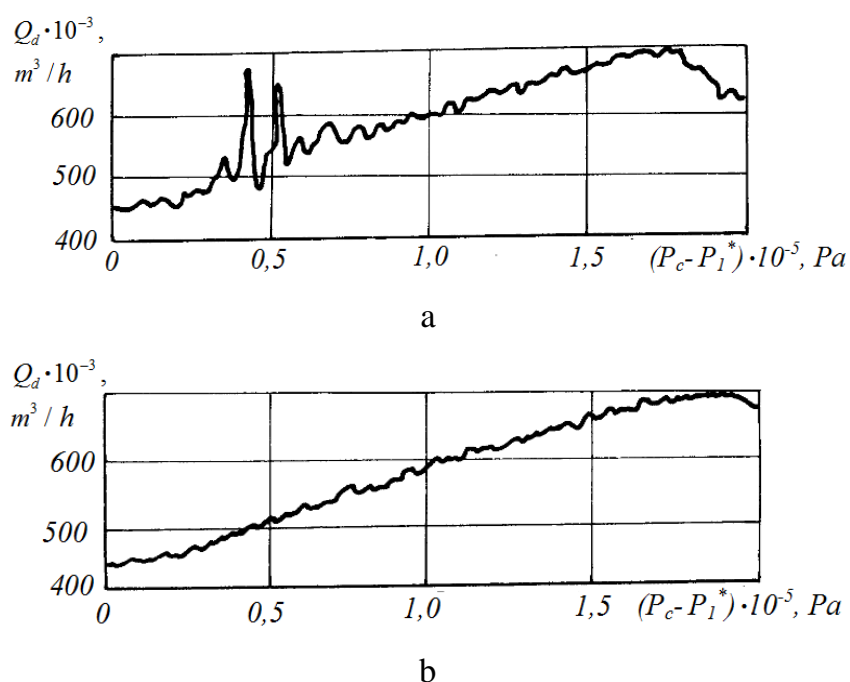
Figure 5: Experimental static performance of engine control system for the altitude starting mode: $a$-standard version; $b$-with silencer in form of acoustic RL-filter at the inlet of the restrictor 7

\section{Conclusion}

The effective method for calculation of static and dynamic characteristics of hydro mechanical control systems under the action of flow ripple is presented. On the example of the turbojet controller it is shown that significant deviation of the engine operating regime can be provoked by changes in performances of resistive elements. To avoid influence of high-frequency fuel flow fluctuations on the engine static performances and to increase system accuracy at the starting mode it is proposed to place the hydraulic $R L$-filter into the control unit. The effect of the device was verified by the engine test results.

\section{References}

[1] K A Edge. Designing quieter hydraulic system - some recent development and contributions. Proc. of the Forth JHPS International Symposium, Japan, Nov., 1999.

[2] V P Shorin. Flow pulsations cancelation in aircraft pipelines. Machine Building, Moscow, 1980. -160 p. (in Russian).

[3] E Kojima \& K A Edge. Experimental determination of hydraulic silencer transfer matrices and assessment of the method for use as a standard test procedure. Proc. Seventh Bath International Fluid Power Workshop, Bath, UK, September 1994, pp. 221-241.

[4] K Lau, D N Johnston \& K A Edge. Fluid borne noise characteristics of hydraulic filters and silencers. Proc. of Seventh Bath International Fluid Power Workshop, Bath, UK, September 1994, pp. 242-261.

[5] V P Shorin et al. Flow ripple and noise reduction in hydro mechanical systems. SSAU, Samara, 2005. -314 p. ISBN 5-93424-203-2 (in Russian). 